\section{Commentary: Can simulation training improve patient outcomes?}

\author{
Ivan Azevedo, $\mathrm{MD},{ }^{\mathrm{a}}$ and Paula Ugalde Figueroa, $\mathrm{MD}^{\mathrm{b}}$
}

Historically, surgical training has followed an "apprenticeship model." The trainee begins operating with a senior surgeon and gradually assumes responsibility for each step of the procedure and the patient's care. In an era with restrictions on working hours, rapidly evolving technology, and fewer residents choosing cardiothoracic surgery as a specialty, surgical education is becoming a more complex endeavor. Training using simulators could transform teaching and the learning process while reducing risk to our patients. The argument for shifting the educational model used for thoracic surgical training toward one that embraces simulation seems strong.

Esophagectomy for esophageal cancer is one of the most challenging procedures performed by surgeons in our specialty, with high rates of morbidity and mortality that are directly related to surgical volume. ${ }^{1}$ Teaching and learning esophageal resection are not easy tasks due primarily to the numerous surgical steps and limited exposure to the anastomosis techniques used in the procedure, which results in a steep learning curve. Although the esophagogastric anastomosis might be considered an easy step, it is a major part of the operation, as it has the greatest long-term impact on comfortable swallowing after surgery. Early and late anastomotic complications are relatively common, with anastomotic leak rates of $10 \%$ to $15 \%$ in modern series. ${ }^{2-5}$ Although the acute complications of an anastomotic leak are usually easily managed, an anastomotic leak frequently results in a chronic stricture, and an operation intended to provide comfortable swallowing might

\footnotetext{
From the a Division of Thoracic Surgery, Department of Oncology Oncobeda, Doctor Beda General Hospital, Rio de Janeiro, Brazil; and ${ }^{\mathrm{b}}$ Department of Pulmonology and Thoracic Surgery, Institut Universitaire de Pneumologie et Cardiologie de Quebec, Quebec, Quebec, Canada.

Disclosures: Authors have nothing to disclose with regard to commercial support.

Received for publication March 26, 2020; revisions received March 26, 2020; accepted for publication March 30, 2020; available ahead of print April 11, 2020.

Address for reprints: Paula Ugalde Figueroa, MD, Institut Universitaire de Cardiologie et de Pneumologie de Québec, 2725, chemin Sainte-Foy, Quebec, Quebec, Canada G1V4G5 (E-mail: paula.ugalde@criucpq.ulaval.ca).

J Thorac Cardiovasc Surg 2020;160:1608-9

0022-5223/ $\$ 36.00$

Copyright (c) 2020 by The American Association for Thoracic Surgery

https://doi.org/10.1016/j.jtcvs.2020.03.139
}

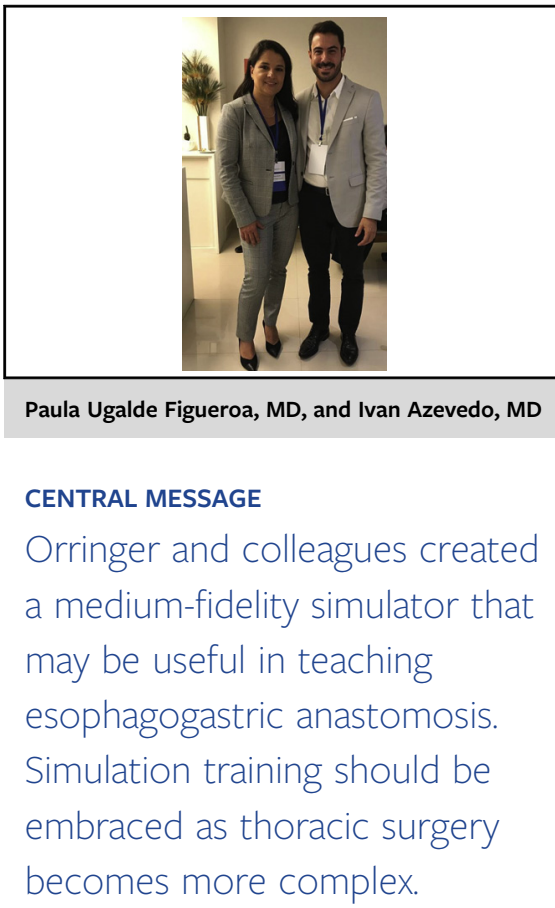

necessitate chronic dilations, ultimately reducing quality of life.

In this issue of the Journal, Orringer and colleagues ${ }^{6}$ describe the design of a cervical esophagogastric anastomosis (CEGA) simulator using 3-dimensional printing technology and provide proof of concept that the stimulator may be helpful in teaching this critical step of transhiatal esophagectomy. After iterative development based on feedback from the senior author, their design incorporated 3 different silicone materials and pigments for softness and tensile strength that mimicked the normal stomach and esophagus. In addition, the simulated esophagus consisted of 2 incompletely fused layers to more accurately mimic the inner esophageal mucosa. Seven faculty and 8 trainees experienced with CEGA from the Section of Thoracic Surgery of the University of Michigan Medical School tested the simulator and completed a 24-item questionnaire to quantitate the fidelity of the simulator. The observed average (OA) of the simulator's fidelity ratings reflected the perceived quality of the stimulator. For the study, an $\mathrm{OA}>4$ reflected the minimally adequate value of fidelity and was documented across all domains queried (realism of materials 4.52 , realism of experience 4.82 , value 4.82 , physical attributes 4.83 , relevance 5.00 ). There were no overall fidelity differences between faculty and trainee ratings. A global rating obtained from a separate survey 
question showed that the evaluators felt that the simulator could be used as a medium-fidelity CEGA training tool $(\mathrm{OA}=3.33$ of 4.00$)$. The data support the model's feasibility, according to self-reported measures of confidence and proficiency and qualitative feedback on the model's fidelity and usefulness. The validity of their findings may be limited by the small size and the origin of the cohort.

The time has come for the thoracic surgery community to embrace training using simulation, as minimally invasive surgery becomes standard of care even for complex procedures. Surgical simulation training does not aim to eliminate the need for interaction with the patient or replace time spent in an operating room but is instead a complement to these experiences to more safely transition to a new medical practice. Models with synthetic material might be considered low fidelity; however, they offer several benefits. They are less expensive to create, are potentially reusable, do not require advanced laboratories, and can be reproduced in large scale. ${ }^{7}$ As we move toward this new model of education, ongoing efforts are needed to document the impact of simulation training on patient safety and clinical outcomes. Although studies have shown cost savings from improving the quality of clinical care, ${ }^{8}$ the costeffectiveness ratio should not overshadow the primary goals of teaching and learning. ${ }^{9}$

This paper provides a nice description of a 3dimensional-printed, silicone-based simulator that will allow trainees to improve their skills in performing the esophagogastric anastomosis necessary for transhiatal esophagectomy. We congratulate the authors on their work thus far and hope both their stimulator and their novel approach to teaching a difficult but crucial step of esophagectomy will improve thoracic surgery training.

\section{References}

1. Birkmeyer JD, Stukel TA, Siewers AE, Goodney PP, Wennberg DE, Lucas FL. Surgeon volume and operative mortality in the United States. $N$ Engl J Med. 2003;349:2117-27.

2. Mishra PK, Shah H, Gupta N, Varshney V, Patil NS, Jain A, et al. Stapled versus hand-sewn cervical esophagogastric anastomosis in patients undergoing esophagectomy: a retrospective cohort study. Ann Med Surg (Lond). 2016;5: 118-24.

3. Saluja SS, Ray S, Pal S, Sanyal S, Agrawal N, Dash NR, et al. Randomized trial comparing side-to-side stapled and hand-sewn esophagogastric anastomosis in neck. J Gastrointest Surg. 2012;16:1287-95.

4. Orringer MB, Marshall B, Chang AC, Lee J, Pickens A, Lau CL. Two thousand transhiatal esophagectomies: changing trends, lessons learned. Ann Surg. 2007; 246:363-72; discussion 372-4.

5. Portale G, Hagen JA, Peters JH, Chan LS, DeMeester SR, Gandamihardja TA, et al. Modern 5-year survival of resectable esophageal adenocarcinoma: single institution experience with 263 patients. J Am Coll Surg. 2006;202:588-96; discussion 596-8.

6. Orringer MB, Hennigar D, Lin J, Rooney DM. A novel cervical esophagogastric anastomosis simulator. J Thorac Cardiovasc Surg. 2020;160: 1598-607.

7. Anastakis DJ, Wanzel KR, Brown MH, McIlroy JH, Hamstra SJ, Ali J, et al. Evaluating the effectiveness of a 2-year curriculum in a surgical skills center. Am J Surg. 2003; 185:378-85

8. Cohen ER, Feinglass J, Barsuk JH, Barnard C, O'Donnell A, McGaghie WC, et al Cost savings from reduced catheter-related bloodstream infection after simulation-based education for residents in a medical intensive care unit. Simul Healthc. 2010;5:98-102.

9. Patel AD, Gallagher AG, Nicholson WJ, Cates CU. Learning curves and reliability measures for virtual reality simulation in the performance assessment of carotid angiography. J Am Coll Cardiol. 2006;47:1796-802. 